\title{
La incorporación de los lectores de prensa impresa a la prensa digital: uso convergente del soporte on-line
}

\section{The incorporation of print media readers to digital media: convergent use of the online platform}

\author{
Iván Lacasa Mas. Universidad Internacional de Cataluña \\ María Victoria Mas. Universidad Internacional de Cataluña \\ Mariano Fernández Díez. Marketing manager de LaVanguardia.com
}

Recibido: 26-VII-2011 - Aceptado: 23-XII-2011

Resumen:

Gracias a una encuesta realizada a más de 3.500 lectores de LaVanguardia.com, el presente artículo sostiene que lo que ha movido a buena parte de ellos a adoptar la lectura de la edición digital no son tanto las especificidades que diferencian a la plataforma on-line, como la confianza que han depositado en su marca periodística. El análisis de los patrones de uso y de las expectativas de dichos lectores lleva a ver el soporte digital no como una superficie de información inmediata, sino como un vehículo flexible de acceso a una fuente tenida como de referencia. Investigar las gratificaciones que obtienen los lectores al establecer una relación con la marca periodística, mueve a trasladar el punto de mira hacia aquello que, por encima de la evolución de los distintos soportes, permanece estable entre los medios de información y sus públicos objetivos.

Palabras clave:

Prensa on-line, usuarios, expectativas, multiplataforma, marca periodística

Abstract:

Thanks to a survey made to more than 3500 readers of La Vanguardia.com, this article holds up that what motivated most of them to adopt the reading of the digital edition was not so much the specific things of the on-line platform but the reliability they have settled on the journalistic brand. The analysis of patrons of use and the expectations of those readers takes us to see the digital support not as a place for immediate information but as a flexible vehicle to access to an information source of reference. The investigation of gratifications obtained by the readers when establishing a relationship with the journalistic brand takes us to move our point of view towards what remains still among media and their audiences, over the evolution of the platforms.

Keywords:

On-line media, users, expectations, multiplatform, journalistic brand

1 El presente artículo surge de un proyecto de investigación sobre la audiencia de la prensa digital realizado en colaboración con el proyecto CSO2009-13713-C05-02. 


\section{Introducción}

Dado el creciente flujo de audiencias hacia Internet, en una época marcada por la crisis económica, el soporte digital y los nuevos dispositivos de recepción son una gran oportunidad para las empresas periodísticas (Fernández, 2011)². No está claro, sin embargo, cómo se va a organizar el ecosistema mediático ni hacia dónde va a evolucionar la relación entre ese nuevo medio y los tradicionales. Las investigaciones realizadas durante la última década se debaten entre sostener que Internet está sustituyendo a los medios clásicos o defender que únicamente los está desplazando, es decir, que está reconfigurando el uso que de ellos se hace (Mitchelstein y Boczkowski, 2010; Nguyen y Western, 2006; de Waal et al., 2005; Lee y Leung, 2006; Dutta Bergman, 2004; Dimmick, 2003; Beaudoin y Thorson, 2002; Robinson et al., 2000; Stempel et al., 2000) ${ }^{3}$. En el caso de las empresas de prensa tradicional, el debate entre esos dos grandes planteamientos se concreta un poco más y desemboca en el esfuerzo por determinar si la lectura de las dos ediciones de un mismo periódico -digital e impresa- evoluciona hacia un tipo de consumo de la digital que complementa al de la impresa o que, por el contrario, compite directamente con ella y está en proceso de sustituirlo.

Según los datos de las oleadas realizadas entre los años 2008 y 2010 por el Estudio General de Medios (EGM) sobre la audiencia de las principales cabeceras de prensa españolas ${ }^{4}$, el número de personas que consultan exclusivamente la versión digital crece; no obstante, también lo hace el número de las que usan ambas ediciones (Gráficos 1-4) ${ }^{5}$. Es entre el grupo de lectores que lee tanto el periódico impreso como el digital donde podría estar dándose un consumo complementario de ambas ediciones, un consumo que -contrariamente a lo que afirmaría la teoría de la sustitución-podría verse como per-

2 Sin ánimo de asentar una terminología propia, pero conscientes de la equivocidad de los términos, denominaremos cabecera a la marca periodística, producto o edición a las distintas versiones de un mismo periódico, y canal, soporte, vehículo o plataforma al medio técnico de distribución o de acceso.

3 No podemos olvidar que la mayoría de las investigaciones que defienden la tesis de la sustitución de los medios tradicionales por el nuevo medio digital han sido formuladas desde un enfoque que podríamos llamar «medio-céntrico» (McQuail, 2000: 30-32). Por contra, aquellos autores que adoptan una perspectiva de tipo «usuario-céntrica» han tendido más bien a concluir que existe una relación complementaria entre ellos (Nguyen y Western, 2006; de Waal et al., 2005; Dutta Bergman, 2004; Stempel et al., 2000). Dicha perspectiva tiende a concluir que, para el usuario, los soportes juegan un papel distinto pero complementario en la consecución de sus propios objetivos (Nguyen y Western, 2006). Nos parece un planteamiento más acertado, no solo por el tipo de relación más flexible que establece entre la pervivencia de los nuevos y los viejos medios, sino porque se centra en el estudio del usuario, al tener una concepción activa de él. La perspectiva usuario-céntrica habla de consumidores que se rigen más por sus dinámicas que por las de los medios, de suerte que sus acciones no pretenden amoldarse a uno u otro medio, sino lograr unos objetivos, dar respuesta a ciertas necesidades o a obtener determinadas gratificaciones mediante ellos (Ruggiero, 2000: 8).

4 A pesar de ser el diario on-line de mayor difusión en España, no incluimos datos de ElMundo.es puesto que dicho diario no está asociado a la AIMC, y el EGM no recoge sus datos (Cfr.: http://www.aimc.es/Sitios-de-Internet.html).

5 Como se puede apreciar en las tablas anexas a los gráficos 1-4 que resumen la variación de lectores para cada cabecera, excepto en el caso de El País, el número de lectores que combinan ambas versiones crece incluso más que lo que disminuye el volumen de lectores de sus ediciones impresas. 
durable si se comprueba que los lectores usan de forma distinta cada soporte, puesto que, en ese caso, la existencia de cada uno de ellos seguiría aportando algún tipo de valor añadido (Fidler, 1997). Si así fuera, la definitiva generalización del acceso a Internet para consultar la prensa no significaría la caída en desuso del periódico impreso, aunque, muy probablemente sí, la reducción del tiempo a él dedicado (Nguyen y Western, 2006). Lo cierto, por el momento, es que el número de lectores de las ediciones impresas todavía es mayor que el de los usuarios de las ediciones on-line (Gráficos 14). Por tanto, será necesario conocer hacia dónde evolucionará la relación que los lectores de una cabecera acabarán estableciendo con cada una de sus ediciones.

Los resultados de uno de los últimos estudios de la AIMC acerca del consumo de prensa impresa y digital puede ayudarnos a entender la relación que con ellas establecen actualmente los lectores: un $76 \%$ de los internautas no solo consulta periódicos en ambos soportes, sino que, en el $57.7 \%$ de los casos, lo que hace es acceder a las dos versiones de una misma cabecera (Gabardo, 2010). Por otro lado, un 76,3\% afirma haber empezado por leer su versión impresa y un $48,4 \%$ responde que "sigue leyendo el periódico impreso como antes" (Gabardo, 2010) ${ }^{6}$. Estos datos sugieren que hoy en España una parte importante de los lectores de prensa digital posee una relación con las cabeceras de prensa tradicional que es anterior al uso de la plataforma digital. Es una relación que han forjado a través de la lectura de la edición impresa y que, por su antigüedad, no puede sino haber generado en ellos ciertos hábitos, valoraciones y expectativas que ahora estarán condicionando también su acceso a la edición digital y el uso que le dan (Livingstone, 1999: 60).

En efecto, tal y como Palmgreen y Rayburn explicaron con el modelo de la expectativa-valor, el uso que la audiencia da a cualquier objeto mediático ${ }^{7}$ depende de la evaluación que hace sobre cómo satisfará sus expectativas ${ }^{8}$ (1985: 63). Además,

6 Se trata de una encuesta realizada a los individuos procedentes del año móvil de la 2a ola del EGM de 2010 que habían accedido a Internet en los últimos 30 días.

7 Palmgreen y Rayburn designan con esta expresión a diferentes realidades, ya sea el propio medio (televisión, radio...), ya sea un programa o cualquier tipo de contenido que se consume a través de ellos (1985).

8 Las gratificaciones buscadas por la audiencia en un objeto mediático dependen de (a) las creencias (probabilidades subjetivas) acerca de las cualidades que posee dicho objeto o sobre el resultado que tendrá un comportamiento relacionado con él, y de (b) las evaluaciones afectivas sobre las cualidades o beneficios particulares de ese objeto. En el caso de que las cualidades de un medio sean percibidas como un componente importante de dicho medio y este sea evaluado positivamente, la teoría predice que habrá una búsqueda intensa de la gratificación a él asociada, si bien dicha intensidad dependerá de las creencias acerca de la relevancia de esa cualidad asociada al medio y de la evaluación que de ella se haga (Palmgreen y Rayburn, 1985: 63-67). Este modelo postula que las expectativas (producto de las creencias) y las evaluaciones influyen sobre la búsqueda de gratificaciones, la cual, a su vez, influye sobre el consumo de los medios, consumo que da lugar a una percepción de ciertas gratificaciones obtenidas, las cuales, entonces, retroalimentan -refuerzan o alteran- las percepciones que un individuo tiene acerca de aquellos atributos que asocia a alguna gratificación de un determinado medio (Palmgreen y Rayburn, 1985: 64). Las evaluaciones del consumo de los medios no dependen de las expectativas, sino que son consideradas elementos estables que son producto de las necesidades individuales y del sistema de valores (Palmgreen y Rayburn, 1985: 65). Las gratificaciones obtenidas no son creencias sino beneficios experimentados (cognitivos, afectivos o conductuales) en el consumo de los medios (Palmgreen y Rayburn, 1985: 66). La complejidad para la definición y operacionalización 
las gratificaciones que de facto vayan obteniendo los distintos usuarios de ese objeto mediático irán determinando el uso futuro que de él harán y la relación que con él acaben estableciendo (McQuail, 2000: 462). El modelo de la expectativa-valor nos invita, por tanto, a pensar que los lectores que provienen de una tradición de prensa impresa habrán obtenido ya unas gratificaciones y generado unas expectativas a través de la lectura de esta, que fácilmente aplicarán a la prensa on-line, sobre todo si proviene de una publicación matriz impresa. Supondremos, por ello, en el presente artículo que el uso que hacen del soporte digital probablemente esté inspirado en el que hacen del soporte papel ${ }^{9}$.

Así, a la hora de estudiar la relación que los lectores están estableciendo con la edición digital de un determinado periódico, será conveniente, en un primer momento, analizar la similitud entre el uso que hacen de ella y el que hacen de su edición impresa. Discernir, en un segundo momento, si las expectativas que les mueven en ese uso atienden principalmente a las especificidades del soporte on-line o, por el contrario, tienen como horizonte aquello que es común en ambas ediciones, permitirá ver si es oportuno afirmar que en el uso de la edición on-line existe una continuidad en la relación ya establecida con la cabecera periodística, relación que, en ese caso, estaría por encima de la complementariedad de ambos soportes y atendería a una percepción unitaria de ambas ediciones. Afirmar ese sentido unitario dado por los lectores a las dos ediciones de su cabecera, implicaría, por último, ampliar la perspectiva del estudio sobre la complementariedad de ambas ediciones al estudio de la relación que establecen los lectores no con el soporte, sino con la cabecera.

\section{Hipótesis y objetivos}

En definitiva, a fin de constatar qué tipo de relación pueden estar estableciendo los lectores con las dos ediciones de una misma cabecera de prensa, partiremos de la siguiente hipótesis: si el uso que hacen de ambos soportes es similar, esa coincidencia se deberá, en primer lugar, a que sus expectativas acerca de la edición digital se asemejan a las que poseen acerca de la edición impresa y, en segundo lugar, a que en lugar de subrayar la especificidad de cada soporte, lo que hacen esas expectativas es denotar una percepción de la lectura de la edición on-line como extensión de la tradición de lectura de la edición impresa.

Así pues, en el presente artículo, nos propondremos los siguientes objetivos:

de conceptos de una naturaleza tan cualitativa como son las expectativas o las gratificaciones ha suscitado múltiples críticas contra el enfoque de usos y gratificaciones, al que pertenece este modelo. A pesar de ello, son también numerosos los autores que hoy siguen defendiendo la validez este enfoque para el estudio del uso de los medios (Ruggiero 2000: 28; Meyen 2004).

9 Hay que tener en cuenta que, durante sus primeros años de vida, la prensa digital que provenía de cabeceras de prensa tradicional se limitaba a ser una mera duplicación de los contenidos que se publicaban en el soporte impreso (Canga et al., 2000: 15; Armentia et al., 2000: 13). Es lógico, pues, que siendo hoy todavía productos informativos bastante similares, ambas ediciones de la prensa on-line generen expectativas semejantes en sus lectores, sobre todo en aquellos que ya consumían la edición impresa de una cabecera determinada y han pasado a leer también su edición on-line. 
Describir de forma relacionada las pautas básicas de consumo de aquellos lectores de una cabecera de prensa que la leen en su edición impresa y digital, para ver si cabe pensar en un uso similar-es decir, no específico- de ellas.

b) Analizar las valoraciones que de la edición impresa y la digital realizan aquellos lectores que acceden a ambas, para confirmar si sus expectativas en el consumo de cada una de ellas son semejantes y para discernir en qué medida aquellas expectativas referidas al periódico digital que coinciden con las del periódico impreso son las que influyen en el acceso y en el modo de consumo particular de la edición on-line.

\section{Metodología}

Como hemos visto más arriba, los datos sobre lectura de prensa en España confirman la existencia de un consumo multiplataforma de los contenidos de una misma cabecera por parte de un sector significativo de los lectores. Este hecho permitiría afirmar que las empresas periodísticas están en buena disposición de mantener una relación estable con muchos de sus lectores a través de más canales que antes, si saben aprovechar las potencialidades técnicas de la Red, ir más allá de ellas y satisfacer las expectativas de uso y relación que tienen los lectores más fieles a la cabecera (McQuail, 1997: 30). El conocimiento de las expectativas que mueven a los lectores cuando consumen información en ambos soportes podría permitir a las ediciones on-line crear una relación estable con un grupo importante de usuarios, potencialmente más comprometidos, como son los lectores ya habituales de la versión impresa. A la vista de esa oportunidad, el presente artículo estudia a los usuarios registrados en LaVanguardia.com ${ }^{10}$, edición digital de una de las cabeceras de prensa de referencia en España, unos usuarios que, como veremos a continuación, mantienen un vínculo con las edición digital e impresa de ese diario.

\subsection{Población y muestra}

Según los datos de la última oleada del EGM de 2010, del total de usuarios de la LaVanguardia.com, un 40\% combina su lectura con la lectura de la edición impresa. Para estudiar a esa población, hemos realizado una extensa encuesta, que tuvo lugar entre los meses de octubre y diciembre de 2010, a 3.593 lectores registrados en LaVanguardia.com ${ }^{11}$.

10 El registro es necesario para participar en foros y blogs, recibir correos electrónicos (newsletters, mailnews...), y suscribirse a la edición impresa. La mayoría de nuestros encuestados estaban registrado para recibir la newsletter o porque eran suscriptores.

11 El porcentaje de abandono fue del $27 \%$ y la duración media de 18 minutos. El $65.77 \%$ de los usuarios que contestaron la encuesta fueron hombres y el 34.23\% mujeres. La distribución por franjas de edad fue la siguiente: $12.51 \%$ la de $26-35$ años, $29.09 \%$ la de $36-45$ años, $29.37 \%$ la de $46-55$ años y $17.55 \%$ la de 56-65 años. Para poder considerar a este grupo de lectores registrados una muestra representativa de todos los lectores de La Vanguardia que combinan la lectura de ambas versiones ha sido necesario equilibrar la muestra, a fin de que la distribución de porcentajes coincidiera con los porcentajes de las franjas de edad establecidas por el EGM. Según esos datos del EGM, los lectores pertenecientes a las franjas de edad de 35-44, 25-34 y 45-54 años son los más numerosos entre la audien- 
Si tenemos en cuenta que el 50,97\% de los encuestados hace más de dos años que conoce LaVanguardia.com y que en el $40,15 \%$ esa antigüedad asciende hasta más allá de los 5 años, bien podemos afirmar que sus patrones de consumo se asientan sobre unos hábitos desarrollados durante un periodo de tiempo considerable, lo cual nos da a entender que los lectores encuestados tienen una relación ya madura con la edición digital, así como con su cabecera periodística ${ }^{12}$. A fin de ajustarnos a los objetivos del presente artículo, limitaremos los datos analizados a las respuestas de aquellos lectores que consumen a diario o muy frecuentemente (2-5 veces a la semana) la edición on-line. Son más de un 70\% de los encuesta$\operatorname{dos}^{13}$. Además, de entre estos lectores habituales de la edición on-line, se han escogido las respuestas de aquellos que leen también la edición impresa, con dos tipos de frecuencia: habitual (a diario o 2-5 veces a la semana) y mínima (una vez a la semana) (Tabla I) ${ }^{14}$.

El análisis de las respuestas de estos dos tipos de perfiles de usuarios, ha ido dirigido a determinar si sus expectativas con respecto a la edición on-line están relacionadas con las expectativas que tienen de la edición impresa y si, por tanto, pueden ser referidas a la cabecera, antes que a las especificidades del nuevo soporte. La división por frecuencias de lectura de la prensa impresa permitirá discernir si el uso más o menos intenso de este soporte tradicional influye en las expectativas de uso y relación con la edición on-line.

cia de LaVanguardia.com que además lee la edición impresa. Cabe destacar que la distribución de edades que hemos obtenido en nuestra encuesta no difiere de la de los lectores que solo leen la edición digital ni -según el EGM- de la de los lectores de la prensa digital española en general. La única desviación respecto al EGM es una pequeño desplazamiento de dos años en los límites que marcan las horquillas de edad de los encuestados. No consideramos que esa diferencia suponga una alteración del valor de los resultados.

12 Para el presente artículo se han seleccionado solo algunas de las 40 preguntas que componían la encuesta completa. No pretendemos hacer una exposición global de los resultados. Queremos centrarnos en una cuestión muy concreta, tal como la hemos presentado más arriba en la introducción, la hipótesis y los objetivos.

13 En cuanto a la experiencia en Internet que tienen los lectores encuestados, podemos afirmar que son usuarios habituales, puesto que el 92,97\% afirma acceder a la Red todos los días. Pasan, además, un tiempo considerable conectados: el 26.63\%, entre 30 minutos y una hora; el $35.22 \%$, entre 1 y 3 horas; el 14.74\%, entre 3 y 5 horas. Casi un $20 \%$ pasa más de 5 horas conectado.

14 No consideraremos una frecuencia de lectura menor -ocasional, en definitiva-suficientemente relevante como para hablar de un vínculo con la cabecera. Nuestro objetivo no es describir a la audiencia de la prensa digital en general, sino fijar la mirada en los lectores con una relación estable con la cabecera, algo que consideramos presumible en las personas que combinan la lectura habitual de un mismo periódico en dos soportes distintos. Estudiaremos las respuestas a la encuesta desde este punto de vista concreto. En el análisis de los datos no tendremos en cuenta las medias generales, calculadas a partir de todas las respuestas obtenidas, sino las medias de aquellos lectores que poseen uno de estos dos vínculos. De todas maneras, los lectores seleccionados siguen siendo la mayoría de los encuestados y en casi todas las cuestiones por las que se les ha preguntado han marcado la media general. El grupo de personas que ha quedado excluido de nuestros análisis, los lectores que compran solo ocasionalmente la edición impresa y leen solo ocasionalmente la edición on-line, representan un 10\% de los encuestados. 


\section{Resultados}

\subsection{Patrones generales de consumo de la prensa impresa y digital}

Tal como hemos visto en la introducción, las investigaciones que se centran en el comportamiento de los usuarios tienden a defender que, en un contexto en el que ya se va extendiendo el uso de los dos soportes de la prensa, papel y digital, las tendencias de consumo de los lectores denotan una relación de complementariedad entre ambos (Nguyen y Western, 2006; de Waal et al., 2005; Dutta Bergman, 2004; Stempel et al., 2000). Según esta perspectiva, ningún medio concreto cuyo uso se haya establecido socialmente puede ser visto como una alternativa funcional absoluta con respecto al resto; cada uno de ellos tiene unas características distintivas para servir a diferentes personas, en diferentes contextos, y así complementar a los otros en la satisfacción de necesidades (Nguyen y Western, 2006).

Si fijamos la mirada en el caso de la prensa tradicional, los datos de consumo en España confirman que una parte importante de los lectores de cada cabecera usa ambos soportes. El estudio de sus pautas generales de consumo debería permitir determinar si los contextos de tiempo y espacio en que esos lectores usan cada soporte son específicos o, por el contrario, coincidentes. Sabríamos así también si las expectativas que pueden estar teniendo acerca de cada una de las ediciones de La Vanguardia son específicas o similares.

\subsubsection{Lugares de lectura}

Como se puede apreciar en la tabla II, los lectores con un vínculo habitual y los lectores con un vínculo mínimo afirman leer la edición impresa y acceder a la edición on-line más habitualmente desde casa que desde el resto de lugares. La práctica más habitual, también con la versión impresa, es leerla en el contexto del hogar. Es ese un lugar de lectura mucho más frecuente que el trabajo o el transporte. Otros lugares de lectura que son también bastante comunes son los bares y cafeterías. Estos datos podrían indicar que la lectura de la prensa impresa está más asociada a los momentos de descanso que la de la edición on-line, ya que el acceso a esta última hoy se suele realizar con más frecuencia desde el ordenador, un dispositivo que se utiliza para el trabajo o cuyo uso podría no ser suficientemente confortable en el hogar.

Sin embargo, en lo que respecta a la lectura de la edición digital, vemos que tanto entre los lectores con un vínculo habitual como entre los lectores con un vínculo mínimo con la edición impresa, el hogar es un lugar de acceso más frecuente incluso que el trabajo, sobre todo en el caso de los lectores con un vínculo mínimo. Puede afirmarse, en definitiva, que hoy el acceso a la edición digital, desde casa o desde el trabajo, no solo es igual de posible desde un punto de vista tecnológico, sino como mínimo igual de real desde el punto de vista del uso que de ellos se está haciendo. 


\subsubsection{Momentos de lectura y tiempo de dedicación a cada edición}

En cuanto a los momentos del día dedicados al consumo de prensa, las personas con un vínculo habitual y las personas con un vínculo mínimo afirman leer la edición impresa y acceder a la edición on-line por las mañanas con mayor frecuencia que por las tardes o por las noches (Tabla III).

No podemos dejar de tener en cuenta, sin embargo, que existe una cantidad nada despreciable de personas que leen ambas ediciones por la tarde y, todavía más, por la noche. En cuanto al uso de la edición on-line, los lectores con un vínculo mínimo con la edición impresa son los que le dedican más tiempo de lectura a la edición on-line por las noches, en vez de por las mañanas. Podemos afirmar, por tanto, que el acceso en uno de los momentos del día menos vinculados al horario laboral, es más común entre los lectores con un vínculo menor con la edición impresa.

Hay grandes diferencias en la cantidad de tiempo dedicado por los usuarios a una y otra edición (Tabla IV). La mayor parte de los lectores, independientemente del vínculo que tengan con la edición impresa, le dedican entre media hora y una hora. También existe un porcentaje menor, pero relevante, de lectores que le dedica menos de media hora. En el caso del consumo de la edición on-line, sucede lo contrario: una gran mayoría de los lectores, sea cual sea su vínculo con la edición impresa, destina un máximo de 30 minutos a la lectura de la versión digital. Entre estos hay casi un $40 \%$ con un vínculo habitual y más de un 50\% de lectores con un vínculo mínimo que invierten menos de 15 minutos.

En definitiva, todos los lectores dedican mucho menos tiempo al uso de la edición digital. Son sobre todo las personas con un vínculo mínimo las que dedican menos tiempo a la edición digital, pero son ellas también las que dedican menos tiempo a la edición impresa ${ }^{15}$.

Una vez visto el tiempo medio que los lectores invierten habitualmente en la lectura de prensa en sus dos soportes, puede ser útil analizar de qué manera varía este tiempo durante el fin de semana. Tal y como se puede apreciar en la tabla V, la gran mayoría de lectores, tanto con un vínculo habitual como con un vínculo mínimo, dedican a la edición impresa más tiempo durante el fin de semana que entre semana. Es un hecho que puede considerarse normal, teniendo en cuenta que durante esos dos días las personas disponen de más tiempo que entre semana y que, además, el producto, sobre todo el domingo, aumenta de paginación y contenidos (Tabla V).

15 El uso menos acentuado de la edición digital responde al mismo tiempo a un perfil de usuarios que tampoco hace un uso frecuente de la edición impresa. Tal y como han demostrado diversos estudios sobre la relación complementaria de ambos soportes, la diversa intensidad de uso de la información on-line está relacionada con el grado de uso de los medios de comunicación tradicionales, especialmente con aquellos que son más intensivamente informativos. Las personas que acuden a Internet para consultar noticias y buscar información siguen utilizando fuentes de información más clásicas. Son lectores con un alto grado de interés por el consumo de información general, independientemente del soporte del que se sirvan para ello (Nguyen y Western, 2006). El énfasis en el consumo de uno y otro soporte depende más del interés del lector por la información que del tipo y características del soporte de que se trate en cada caso.

\section{0 | n 14 | doxa.comunicación}


Si nos centramos ahora en la variación de la cantidad de tiempo que los usuarios dedican a la edición digital durante el fin de semana, comprobaremos que, en ambos grupos, casi un 50\% dedica el mismo tiempo o más a la edición on-line durante esos días, mientras que el otro $50 \%$ dedica menos o nada de tiempo.

Al comparar los hábitos de los lectores con un vínculo habitual con los de los lectores con un vínculo mínimo, percibimos que estos segundos dominan entre los encuestados que afirman dedicarle más tiempo a la edición on-line el fin de semana. En cambio, entre los que afirman dedicarle el mismo tiempo, los lectores que tienen un vínculo habitual son los que superan a aquellos que tienen un vínculo mínimo, sin que estas variaciones del tiempo dedicado por unos y otros lectores llegue a representar nunca una diferencia radical.

Finalmente, casi el 80\% de las personas que leen la edición impresa durante el fin de semana invierte más tiempo en esa actividad entonces que entre semana ${ }^{16}$. Al menos en la mitad de los casos ello no implica un desplazamiento de la edición digital, ya que casi un 50\% de los encuestados le dedica el mismo tiempo o más a la edición digital durante el fin de semana.

\subsubsection{Conclusiones parciales}

En resumen: tras haber estudiado los patrones generales de consumo de aquellas personas que leen de forma habitual las dos ediciones de La Vanguardia, podemos sostener que los contextos físicos o temporales desde los que acceden tanto a la edición impresa como a la edición on-line, son similares. Por un lado, faltan indicios de que una lectura generalizada de la edición impresa en casa o en otros lugares no relacionados con la actividad laboral implique que el uso que los lectores hacen de la edición digital tenga lugar preferentemente en el trabajo. Por el otro, el hecho de que las mañanas sean el momento del día en que usan de forma generalizada tanto una versión como la otra indica que la lectura de una no excluye la lectura de la otra en el mismo momento del día.

Los datos de consumo de aquellos días más vinculados al ocio y al descanso tampoco confirman una reconfiguración del modo de uso de una versión debido al consumo de la otra: el predominio de la prensa impresa durante esos dos días no se traduce en un monopolio, pues en ningún caso puede decirse que ese fenómeno coincida con una marcada reducción

16 El 45,77\% de los lectores que mantienen un vínculo habitual con la edición impresa afirman comprarla solo el fin de semana. En cambio, entre los lectores con un vínculo mínimo, solo hay un $24.57 \%$ que dicen adquirirla exclusivamente el fin de semana. Por su parte, los encuestados que afirman leer la edición impresa tanto entre semana como el fin de semana son un $46.81 \%$, en el caso de los lectores con un vínculo habitual, un $67.97 \%$, en el caso de los lectores con un vínculo mínimo. Las personas con cualquiera de los dos vínculos que leen la edición impresa exclusivamente entre semana son una clara minoría: en torno al 7\%, en ambos casos. Por otro lado, la mayoría de lectores que compran semanalmente la edición impresa, pueden hacerlo tanto entre semana como el fin de semana. Cabe afirmar, en definitiva, que la costumbre de leer la edición impresa durante el fin de semana está muy extendida, aunque no es exclusiva de esos dos días, puesto que también es alta la frecuencia con que se lee entre semana. Además, a pesar de que la lectura de la edición impresa se asocie más a contextos de ocio o descanso, los lectores que mantienen un vínculo mínimo con ella pueden decidir leerla tanto en días festivos como laborables. 
general del tiempo dedicado a la edición on-line. Por último, tal como veremos con más detalle en el apartado siguiente, el hecho de que ya de forma permanente se le dedique menos tiempo al soporte digital que al papel parece más relacionado con el uso estructuralmente asociado a ese soporte que con un fenómeno de competencia cuantitativa entre el papel y lo digital.

En definitiva: no existen evidencias de que las relaciones que los lectores estudiados mantienen con cada una de las ediciones de La Vanguardia entren en competencia, aunque tampoco queda demostrado que sean complementarias. La coincidencia en los patrones generales de consumo podría estar denotando, más bien, una percepción similar de ambos soportes y, por tanto, unas expectativas también semejantes acerca de su uso. Será necesario, pues, estudiar las expectativas que influyen en el consumo de una y otra edición, comprobar hasta qué punto coinciden y hasta qué punto tienen un reflejo en el uso que los lectores dan a la plataforma on-line. Solo así será posible determinar el sentido que otorgan al consumo de ambos soportes y apuntar hacia dónde es probable que evolucione la relación que están estableciendo con las dos ediciones de su cabecera de referencia.

\subsection{Expectativas para las dos ediciones y uso específico de la edición on-line}

La difusión de un nuevo medio debe ser entendida no solo como un proceso de mercado, sino también como un proceso social, en el que los significados y las prácticas que finalmente van cristalizando en torno a él tienen trayectorias difíciles de prever porque son fruto de una interacción entre factores tales como los usos actuales y previstos, las representaciones culturales o los intereses individuales (Livingstone, 1999: 60). En el caso de la prensa impresa, ese proceso ya ha tenido lugar. De ahí que el estudio del proceso de difusión e implantación de la prensa digital no pueda dejar de tener en cuenta el hecho de que, en muchas ocasiones, ella proviene de una edición impresa. Probablemente eso implique que muchos de los lectores adopten la lectura de la edición on-line habiendo desarrollado ya un conjunto de valoraciones y expectativas tanto acerca de lo que debe ser un periódico, como de lo que representa su marca periodística de referencia.

Para una verdadera comprensión del uso de la versión on-line que realizan los lectores que mantienen una relación con una cabecera periodística, resulta útil no solo profundizar en las principales valoraciones que hacen de la joven edición digital, sino compararlas con las que tienen de la edición impresa. Ello permitirá determinar hasta qué punto coinciden esas valoraciones y expectativas referentes al uso de los dos soportes y qué influencia tienen en el acceso al soporte on-line, así como en el tipo de lectura específica que de la edición digital hace este tipo de lectores.

\subsubsection{Valoraciones de la edición impresa y de la edición on-line}

En lo que se refiere a la versión impresa y los valores que definen lo que debe ser un periódico, las afirmaciones con las que se identifican los lectores con uno y otro tipo de vínculo son muy similares (Tabla VI). "Me gustan los reportajes a fondo 
que analizan un tema en concreto", "Lo más importante en el periódico es que sea actual”, "Compro La Vanguardia porque es más objetiva que otras cabeceras" y "me identifico con la línea editorial de La Vanguardia” son las afirmaciones con mayor grado de acuerdo.

De las seis primeras más valoradas, la segunda, la quinta y la sexta sentencia de esta tabla trazan de forma clara el perfil del periódico impreso que los lectores esperan encontrar. Para ellos, un periódico es algo que tiene que ser "actual", que es "más objetivo y veraz que otros medios de información" y que no tiene por qué ser "entretenido y divertido". No es extraño, por tanto, que la afirmación con la que más se identifiquen sea la que valora la profundidad de los análisis. Tampoco lo es que, siendo lectores habituales de La Vanguardia, trasladen los valores que acabamos de señalar a la marca periodística y la definan como objetiva, tal y como puede verse en la tercera afirmación de la tabla.

Siendo las anteriores las principales expectativas acerca de la edición impresa, se entiende que las afirmaciones referidas a las columnas de opinión y a la función de entretenimiento no ocupen un lugar preferente en la mente de los lectores, quienes tampoco se identifican con aquellas afirmaciones que denotarían una lectura superficial del periódico, como, por ejemplo, ojear los titulares o no leer a fondo las noticias. Lo mismo sucede con el hecho de comprar el periódico por motivos que denotan poca frecuencia o una baja valoración del producto periodístico. Como muestran las respuestas de los encuestados, las afirmaciones menos valoradas son "compro La Vanguardia porque lleva un suplemento interesante" o "compro La Vanguardia cuando me interesa un tema concreto".

De hecho, con respecto a la versión digital (Tabla VII), la sentencia con la que se identifican más lectores es "Aunque leo la versión digital, me gusta más el periódico de papel". Hay que destacar que los encuestados que mantienen un vínculo mínimo con el diario impreso son los que manifiestan un mayor grado de acuerdo con esta afirmación, lo cual indica que leer con poca frecuencia la edición en papel no implica una menor valoración de este soporte, ni de los ideales que encarna. Además, al ser esta la única afirmación a la que ambos tipos de lectores se adhieren con claridad, cabe verla como una clave de interpretación para el resto de valoraciones, las cuales despiertan en ellos una adhesión débil, y están mucho más asociadas a funcionalidades o características específicas de la edición on-line (su puntuación se sitúa siempre en torno al 3) ${ }^{17}$.

Las valoraciones que comparten los lectores con ambos tipos de vínculo y que destacan por encima de los 3 puntos son aquellas que tienen que ver con la accesibilidad, la inmediatez y la actualidad de la información: "La versión digital es de más fácil consulta”, "Prefiero LaVanguardia.es porque aporta información más actualizada que la versión en papel” y "Leo la versión digital porque es gratis". No obstante, el hecho de que estas afirmaciones no cuenten con un apoyo que resulte significativo induce a pensar que las características de las que hablan no son esenciales para la elección y el uso de la edición digital. Ejemplo de ello es la valoración que recibe por parte de los lectores que tienen un vínculo mínimo la afirmación

17 En una puntuación del 1 al 5, el 3 corresponde a una opción que denota más bien indiferencia o indefinición. 
"Leo tanto el papel como el digital porque son cosas distintas": aunque es la que destacan en segundo lugar, con la puntuación que le otorgan demuestran no establecer una diferencia radical entre una y otra edición, lo cual, a su vez, explica que tampoco le concedan excesiva relevancia a aquellas funcionalidades técnicas del soporte on-line que le distinguen del soporte papel.

Por su parte, las afirmaciones que constituyen comparaciones entre características del soporte tradicional y el digital revelan que ambos tipos de lectores valoran la edición on-line de forma muy similar a la edición impresa. Según los datos que mostraba la tabla VI, comentada más arriba, la confianza y la objetividad, dos de las características primordiales que debe reunir un periódico, son atribuidas por los usuarios también a la edición on-line, en la medida en que, a su juicio, esta no las posee en un grado menor que la impresa (Tabla VII). Pese a que algunos tópicos sobre la prensa on-line sostienen que tanto la confianza como la objetividad podrían verse afectadas por la inmediatez que permiten las condiciones técnicas del soporte digital, el análisis de las percepciones de los lectores habituales de ambas ediciones indica que, de hecho, juzgan de modo similar la edición on-line y la impresa: no se identifican con la afirmación que concede más confiabilidad a la edición impresa y, además, otorgan a la edición on-line el mismo grado de objetividad que le otorgaban a la edición en papel.

Finalmente, las funciones relacionadas con el entretenimiento y la participación, que en la versión on-line, gracias a su dimensión multimedia e interactiva, podrían tener más peso en las expectativas de uso de los usuarios, reciben una valoración baja, igual que sucedía en el caso del periódico impreso.

\subsubsection{Uso de la edición on-line: funcionalidades más utilizadas}

Las valoraciones de los lectores que hemos estudiado en el punto anterior quedan confirmadas ahora con sus respuestas acerca de las funcionalidades que más utilizan del soporte on-line. Tal como puede apreciarse en la tabla VIII, las hemos organizado según tres grandes categorías que responden a las principales funcionalidades que los estudios del uso de los medios digitales de información han definido: búsqueda de información actualizada, búsqueda o recuperación de información específica, y participación (Flavián y Gurrea, 2006; Chung, 2008; Chung y Yun Yoo, 2008; Ruggiero, 2000).

Existen varias diferencias de comportamiento en el uso de la versión on-line entre los lectores que tienen un vínculo habitual con la edición impresa y los que mantienen un vínculo mínimo con ella (Tabla VIII). Por una parte, los lectores con un vínculo habitual tienen un mayor grado de actividad en la categoría de búsqueda de información actualizada. Por otra, los lectores con un vínculo mínimo manifiestan ser más activos en la categoría de búsqueda de información específica. Mientras para los lectores con un vínculo habitual la suma de porcentajes de las tres primeras preguntas de la tabla 4 supera en un $40 \%$ a la de los lectores con un vínculo mínimo, para estos últimos la suma de porcentajes de las cuatro preguntas siguientes supera también en cerca de un $40 \%$ a la de los lectores con un vínculo habitual.

$24 \mid n^{\circ} 14$ | doxa.comunicación 
Por otra parte, es oportuno destacar que en los usuarios con un vínculo habitual, la primera categoría (búsqueda de información actualizada) prácticamente dobla a la segunda (búsqueda o recuperación de información específica). Por el contrario, en los usuarios con un vínculo mínimo ambas categorías tienen porcentajes similares y, dentro de ellas, una distribución homogénea. Son datos que podrían estar indicando que el interés de los lectores con un vínculo habitual está más focalizado hacia la información de actualidad general que el de los lectores con un vínculo mínimo, los cuales, por su parte, parecen tener intereses más variados y específicos.

No cabe olvidar, sin embargo, que entre las actividades más realizadas por ambos tipos de lectores la que coincide es "consultar información de última hora”. A pesar de las diferencias observadas entre ambos grupos, la inmediatez y la actualización constante del medio digital son las dos funcionalidades que todos los lectores habituales del soporte online, independientemente de cuál sea su vínculo con el impreso, suelen utilizar con más frecuencia, a pesar de que ese no sea el motivo fundamental de su elección del soporte digital, tal y como hemos visto en el punto anterior.

Resulta significativo, asimismo, que los usuarios con un vínculo habitual que aseguran 'leer los titulares de la portada' sean un $30 \%$ más que los usuarios con un vínculo mínimo que dicen hacerlo también. Estos últimos afirman tener como actividad principal 'consultar información de última hora'; menos de la mitad de ellos dice leer los titulares de la portada ${ }^{18}$. Es un comportamiento aparentemente paradójico, dado que la lectura de los titulares de la portada suele asociarse al consumo de información de última hora. La explicación puede radicar en que los lectores con un vínculo mínimo -y, por tanto, con poca frecuencia de compra del periódico impreso- acceden a la versión on-line más interesados en noticias específicas, un tipo de consumo ligado a tiempos de dedicación más breves, propios de estos lectores, según hemos visto en el apartado anterior. Este interés de los lectores con un vínculo mínimo supera en grado al interés que manifiestan los lectores con un vínculo habitual por adquirir la perspectiva general de la actualidad que proporciona la portada de un periódico, ya sea en la versión on-line o en la impresa.

Por último, existe un paralelismo absoluto entre las actividades menos realizadas por ambos tipos de usuarios. Sus porcentajes de realización de actividades relacionadas con la participación y la interacción con otros lectores son bajos y las diferencias entre los usuarios con un tipo u otro de vínculo nunca superan el $6 \%$ en este campo.

18 Como hemos visto, los lectores con un vínculo mínimo son también los que afirman dedicarle menos tiempo tanto a la lectura de la edición impresa como a la lectura de la edición on-line. Es un dato que nos mueve a concluir que estamos ante un perfil de consumidor de medios menos intensivo que aquel que mantiene un vínculo habitual con la edición impresa. Su preferencia por la información específica y de última hora, antes que por una visión general de la actualidad, parece confirmar esta idea. 


\subsubsection{Conclusiones parciales}

Si tenemos en cuenta que los dos factores por los que el público suele conceder credibilidad periodística a una fuente son, por un lado, la percepción de competencia profesional y, por el otro, la percepción de integridad, bien podemos afirmar que los lectores encuestados consideran la edición impresa de La Vanguardia una fuente de información creíble (Oyedeji, 2007: 118; Kiousis, 2001: 383). En primer lugar, las valoraciones que los lectores de la edición impresa de esa cabecera hacen, indican que la tradición de lectura de prensa en ese soporte ha generado en ellos ciertas expectativas acerca de lo que en general debe ser un periódico: una institución que aporte información actual, objetiva, creíble y fundamentada. En segundo lugar, los lectores encuestados trasladan estos valores a la cabecera que han escogido como de referencia: afirman leer La Vanguardia porque es más objetiva que el resto y dicen identificarse con su línea editorial.

A su vez, es necesario recordar que la credibilidad global otorgada a un medio de comunicación es el producto de la credibilidad otorgada a su marca periodística, a sus profesionales y a cualquiera de los medios de difusión a través de los que difunde sus contenidos (Oyedeji, 2007; Bucy, 2003; Kiousis, 2001). Por lon tanto, cuando las personas encuestadas otorgan credibilidad a La Vanguardia están otorgándosela, en mayor o menor medida, a esos tres elementos. De ahí que podamos afirmar, pese a los pocos años transcurridos, que los lectores extienden al soporte digital la misma credibilidad y confianza que, tras años de lectura de la edición impresa, otorgan a la marca periodística. Es un fenómeno que se verifica en el hecho de que, aunque la mayoría de encuestados manifieste una preferencia general por el periódico impreso, eso se debe a que depositen una mayor confianza en el soporte papel, pues a la edición digital tampoco le niegan uno de los mayores valores que ellos mismos otorgan a su marca periodística: la credibilidad basada en la objetividad ${ }^{19}$. Cabe concluir, por tanto, que el motivo principal del acceso a la versión on-line, al menos en el caso de las personas que leen las dos versiones de La Vanguardia, es básicamente el mismo que el motivo de lectura de la versión impresa: la necesidad de informarse, y de hacerlo mediante una fuente que les parece digna de confianza. De hecho, tal como hemos visto, este tipo de usuarios habituales de la edición on-line no la elige porque la considere algo radicalmente distinto a la edición impresa.

Las expectativas que les conducen al modo que tienen de leer la edición digital son las mismas que mantienen tanto en relación a la marca como en relación a su visión de qué es y debe ser un periódico: más allá de las potencialidades netamente interactivas y participativas que diferencian a la plataforma digital, la accesibilidad y la inmediatez de la información de actualidad general son las características que alimentan las expectativas de gratificación específicas que se asocian al soporte on-line. A la vista de las respuestas de los lectores encuestados, estos valores asociados a las especificidades del soporte no

\footnotetext{
19 Sigue abierto un gran campo de investigación: determinar por qué muchos de los lectores que usan habitualmente la versión digital y le otorgan los mayores valores periodísticos siguen manifestando una preferencia por la versión impresa. No es posible todavía afirmar si el motivo está relacionado con las costumbres o rutinas ya adquiridas por los lectores actuales de prensa on-line, con factores emocionales o con las funcionalidades del soporte papel y de los formatos a él asociados.
}

\section{6 n 14 | doxa.comunicación}


pueden ser considerados motivos de peso en la elección de la edición digital. Sí son, por el contrario, gratificaciones asociadas a las funcionalidades distintivas del soporte on-line, cuya búsqueda influye en el modo en que los lectores utilizan estas últimas. Muy por encima de las actividades relacionadas con la mayor participación que permite la edición digital, los lectores afirman realizar actividades dirigidas, en primer término, a un consumo más inmediato y directo de información de actualidad general y, en segundo término, a la recuperación y consulta ágiles de información específica.

En definitiva: retomando la hipótesis planteada al inicio del presente artículo, podemos confirmar que algunas de las expectativas más importantes que mueven al consumo de ambos soportes están relacionadas entre sí, ya que miran a la identidad periodística de ambos soportes y no a sus especificidades en tanto que plataformas de difusión de los contenidos de una misma cabecera. Así lo confirma el uso del soporte digital que hacen los lectores encuestados, al ser análogo a aquel que hacen del soporte papel: se asemeja en el objetivo final, informarse; se diferencia en que responde a un consumo más flexible y breve.

\section{Conclusiones finales}

Gracias a una encuesta realizada a un amplio grupo de lectores de La Vanguardia que habitualmente lee su edición digital y su edición impresa, el presente estudio aporta una nueva perspectiva a la investigación sobre la incorporación de los lectores de prensa impresa al soporte on-line. A lo largo de estas páginas hemos podido comprobar que lo que mueve a adoptar la lectura de la versión digital a buena parte de las personas encuestadas no son las especificidades que la diferencian de la impresa, sino la confianza que ya han depositado en su marca periodística, gracias a la relación que han establecido previamente con esta última a través de la lectura de la edición en papel.

Tanto los lectores con un vínculo habitual como los lectores con un vínculo mínimo con la edición impresa de La Vanguardia presentan patrones de consumo muy similares de ambas ediciones de esa cabecera. Esa semejanza observada en el uso del soporte impreso y digital, nos lleva a afirmar que los dos grupos de lectores le dan un sentido unitario al uso de ambos. Puesto que, a pesar de sus diferencias en la frecuencia de lectura de la edición impresa, no se han observado diferencias radicales en los patrones de unos y otros lectores, concluimos que ese sentido unitario no está tan relacionado con la intensidad de uso del soporte impreso como con lo que representa para los lectores la marca periodística ${ }^{20}$. Aunque hayan forjado su relación con la cabecera a través de la lectura de la edición impresa, en un contexto de consumo de ambos

20 Como hemos explicado ya, entendemos que la mayor o menor frecuencia de compra de la edición impresa, así como las leves diferencias observadas en el tiempo dedicado a cada una de las ediciones por ambos tipos de lectores, dependen en muchos casos del tipo de perfil de consumidor, ya de por sí más o menos interesado por la información o con más o menos disponibilidad para consumir los contenidos de su cabecera. En términos de relación con la marca, podemos afirmar, por tanto, que ambos tipos de lectores se comportan de forma similar, aunque su intensidad de uso de cada medio sea distinta. 
soportes, puede afirmarse que la relación principal que los lectores de ambas ediciones han establecido es con aquello que les otorga unidad y está por encima de ellas: la marca periodística. Lo hemos podido confirmar mediante el análisis de las expectativas de los lectores referentes a ambas ediciones y del uso específico que hacen del soporte on-line.

En sus respuestas acerca de la edición impresa, los lectores demuestran haber establecido expectativas acerca de lo que debe ser un periódico, expectativas que extienden a la marca periodística que han escogido como de referencia: La Vanguardia. Si hay algo que las personas encuestadas destacan con mayor rotundidad son valores como la actualidad, la objetividad y la veracidad, que reconocen en su marca periodística, a la cual dicen elegir por ser más objetiva que otras y con cuya línea editorial dicen identificarse. En cambio, las valoraciones que hacen de la edición digital demuestran que sus expectativas referentes a las características específicas del soporte on-line no están todavía muy definidas. Aquellas que sí dicen tener los lectores son básicamente una mayor actualización de las noticias y un acceso más fácil a la información de su cabecera. Sin embargo, estas expectativas no pueden ser consideradas motivos claros para el acceso al soporte online, dado que, aun siendo las características que más valoran, las puntúan con indiferencia. No obstante, esa indiferencia no debe ser interpretada como un desapego del soporte on-line, puesto que, a pesar de la tibieza de sus respuestas, son lectores habituales de la edición digital y-como hemos visto también-le otorgan la misma confianza que a la edición impresa, aunque a esta última hoy reconozcan tenerleeeee más aprecio que a la digital.

A pesar de la poca rotundidad con que los encuestados manifiestan sus expectativas referentes a la edición on-line, el modelo de expectativa-valor sugiere que una manera de avanzar hacia el conocimiento de estas es el uso que el público le da a un objeto mediático (Palmgreen y Rayburn, 1985: 63). Atendiendo a esa idea, si acudimos a las respuestas de nuestros lectores que se refieren al uso que hacen de la edición on-line, comprobamos que sus principales actividades giran en torno a la consulta de información de actualidad general, siempre de una manera más directa y ágil que mediante la edición impresa. Otras de sus actividades más frecuentes son las relacionadas con la búsqueda o recuperación de información específica. Todo ello sugiere que lo que deben de estar encontrando en el soporte on-line -y que va camino de convertirse en una expectativa consciente, poderosamente asociada a él- es una oportunidad para un consumo más flexible de los contenidos de su cabecera. En definitiva, tras haber visto que los lectores encuestados comparten la mayor definición de las expectativas referentes a lo que debe ser un periódico y su marca periodística que comparten los lectores encuestados, podemos concluir que lo que el uso de la edición on-line les permite es sobre todo satisfacer de modo más fácil una expectativa que sí puede ser considerada central para la elección del soporte digital: acceder a una fuente informativa que consideran creíble, con la que ya mantienen una relación y se identifican. El contacto con dicha fuente es la expectativa que les mueve a usar los dos soportes, también el digital, más aún si este último les ofrece un acceso rápido y fácil a la información²1.

21 Se ha señalado que aquello en lo que el medio digital supera a los tradicionales es sobre todo en su capacidad de satisfacer un mayor número de oportunidades de gratificación relacionadas con la amplitud de posibilidades de acceso a los contenidos o con una mayor 
Aunque, tal como hemos visto en la introducción, el debate general sobre la convivencia de los nuevos y los viejos medios se plantee de ordinario en términos de complementariedad frente a sustitución, en el caso de las dos ediciones de una cabecera de prensa vemos conveniente ampliar esa perspectiva. Aun siendo una evidencia que la difusión de las dos ediciones se realiza a través de dos medios distintos, digital uno, impreso el otro, tratándose en realidad de dos versiones de una misma cabecera, el sentido que los lectores otorgan al uso que de ambas hacen es unitario, es decir, fruto de la percepción no de estar haciendo dos cosas básicamente distintas, sino una sola: acceder -independientemente de por qué vía- a la información de actualidad que ofrece una misma fuente informativa de su preferencia.

Ignorar ese hecho y centrar el discurso en la diferencia entre plataformas significaría trabajar con una idea poco exacta del sentido que tiene la evolución actual de los modos de consumo. Los lectores aquí estudiados tienen una relación con La Vanguardia y leen sus dos ediciones, no porque las plataformas que dan acceso a ellas les permitan informarse de forma heterogénea, como si fueran dos espacios de información independientes, sino porque, en su multiplicidad, les sirven como vehículos que facilitan un acceso, distinto pero convergente, a la información ofrecida por aquella cabecera que para ellos es una fuente informativa de referencia.

Dicho de forma más aguda: para el lector habitual, la marca, no el soporte, es la auténtica fuente de información. Las distintas ediciones de una misma cabecera de prensa son relevantes para él no tanto por la especificidad de su soporte, como por constituir oportunidades adicionales de acceso a los contenidos de su cabecera de referencia. Vistos desde esa perspectiva, los diferentes medios usados por las cabeceras periodísticas como plataformas para sus diferentes ediciones, no compiten en la mente del lector, sino que adquieren importancia por aquello común hacia lo que conducen, es decir, en cuanto que aumentan el número y tipo de oportunidades de acceso a los contenidos que ofrece una cabecera periodística determinada. Lo que el tipo de lector que hemos estudiado busca como específico cuando emprende la lectura de prensa es la marca; a lo que accede con un sentido algo más genérico es a la plataforma.

adaptación a los patrones temporales de consumo de sus usuarios (Dimmick 2003: 104). Aunque, debido a sus características propias, el soporte digital puede responder con mayor eficacia que los soportes tradicionales a las distintas necesidades de información, entretenimiento e interacción de sus usuarios, se diría que, al menos por el momento, y entre el público aquí estudiado, no debe su éxito tanto a la satisfacción de necesidades como a la generación de gratificaciones. En un entorno de máxima competitividad entre los medios de información, los usuarios cuentan con innumerables opciones para cubrir sus necesidades básicas de información. Es razonable, pues, pensar que cuando existe una oferta abundante, el hecho de obtener gratificaciones pese más que el hecho de cubrir necesidades. Por otro lado, sabemos que las gratificaciones que los usuarios obtienen de la relación con un objeto mediático cualquiera no solo están relacionadas con la conducta a la que el uso del soporte conduce, sino también con satisfacciones cognitivas y afectivas. En nuestro caso, estas últimas se referirían a la relación con la marca periodística (cfr. Palmgreen y Rayburn, 1985:66; Dimmick 2003: 94-104). Entendemos, por tanto, que lo que mueve al uso y adopción de la edición on-line de una cabecera de prensa tradicional es sobre todo la mayor facilidad o flexibilidad de acceso a los contenidos de una determinada cabecera periodística que ofrece, si bien sostenemos que lo que determinará la consolidación de una relación estable entre medio y audiencia será una identificación con esa cabecera periodística que se afiance a través del consumo de sus distintos productos. 
Ciertamente, en un contexto en el que las audiencias son cada vez más numerosas y sus miembros menos predecibles, los medios tienen cada vez más dificultades para identificar o retener a sus audiencias propias (McQuail, 1997: 23). Frente a esa fragmentación, afirmar la existencia de una relación con las cabeceras periodísticas y estudiarla implica, tal y como hemos visto, profundizar en algo que permanece estable en la relación entre las empresas periodísticas y sus audiencias, por encima de la evolución y multiplicación de los distintos soportes a través de los que será posible acceder a sus contenidos. En una circunstancia de tan alta competitividad entre los medios informativos como la actual, las distintas plataformas pueden servir, además de para ofrecer contenidos, para facilitar el que los lectores vayan afianzando su relación con las cabeceras periodísticas. Si el proceso de convergencia en la que la industria mediática se halla inmersa se orienta hacia la distribución multiplataforma de contenidos (Cabrera, 2010), las empresas periodísticas no deberían descuidar el estudio de aquellas expectativas concretas que sus públicos objetivos tienen acerca de la relación con los medios y con sus marcas periodísticas, por encima de las expectativas de uso de cada uno de sus soportes o dispositivos. Solo entendiendo el sentido que invierten los lectores en el uso de cada uno de sus vehículos de acceso lograrán mantener una relación estable con sus audiencias (Callejo, 2001: 99). El soporte on-line puede representar una gran oportunidad para las cabeceras de prensa, siempre que a través de él sepan afianzar o ampliar una relación que, de hecho, ya mantienen con muchos de sus lectores.

\section{Tablas y gráficos}

Gráficos 1-4. Variación de la lectura de cada soporte en las principales cabeceras de prensa españolas según datos del EGM

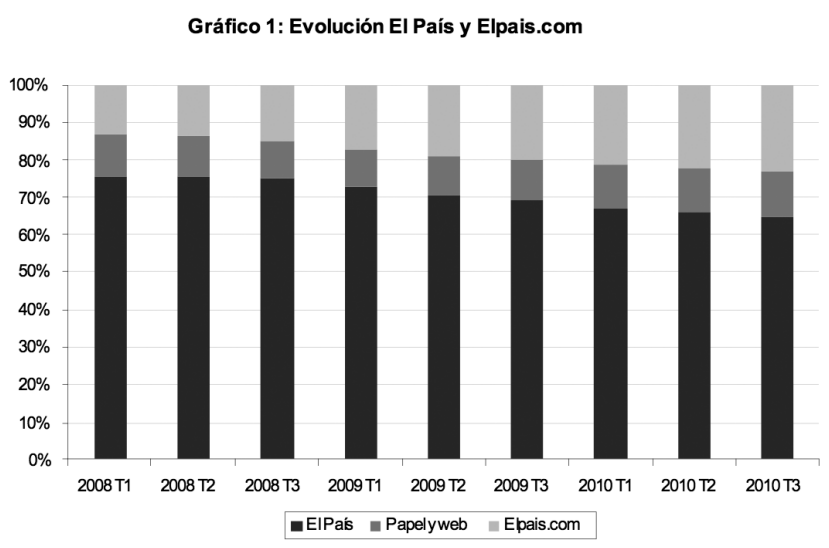

\begin{tabular}{|l|l|}
\hline & Variación 2008 \\
\hline El País & $-14,11 \%$ \\
\hline ElPaís.com & $41,63 \%$ \\
\hline Leen ambos & $3,90 \%$ \\
\hline
\end{tabular}


Gráfico 2: Evolución ABC y ABC.es

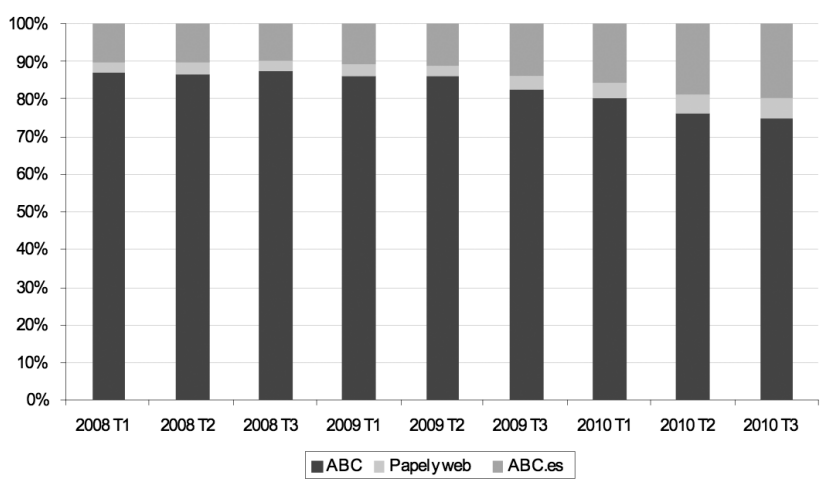

\begin{tabular}{|l|l|}
\hline & Variación 2008 \\
\hline ABC & $6,97 \%$ \\
\hline ABC.es & $128,76 \%$ \\
\hline Leen ambos & $113,64 \%$ \\
\hline
\end{tabular}

Gráfico 3: Evolución La Vanguardia y LaVanguardia.es

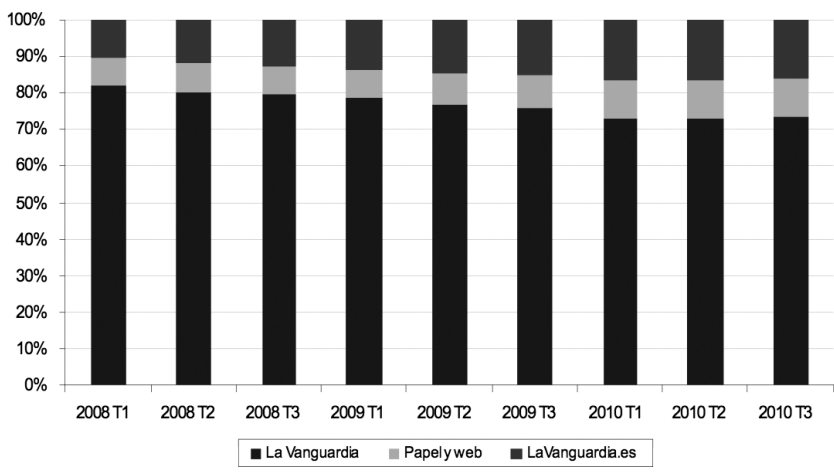

\begin{tabular}{|l|l|}
\hline & Variación 2008 \\
\hline LaVanguardia & $2,07 \%$ \\
\hline LaVanguardia.es & $61,74 \%$ \\
\hline Leen ambos & $48,33 \%$ \\
\hline
\end{tabular}

Gráfico 4: Evolución El Periódico de Catalunya y Elperiodico.com

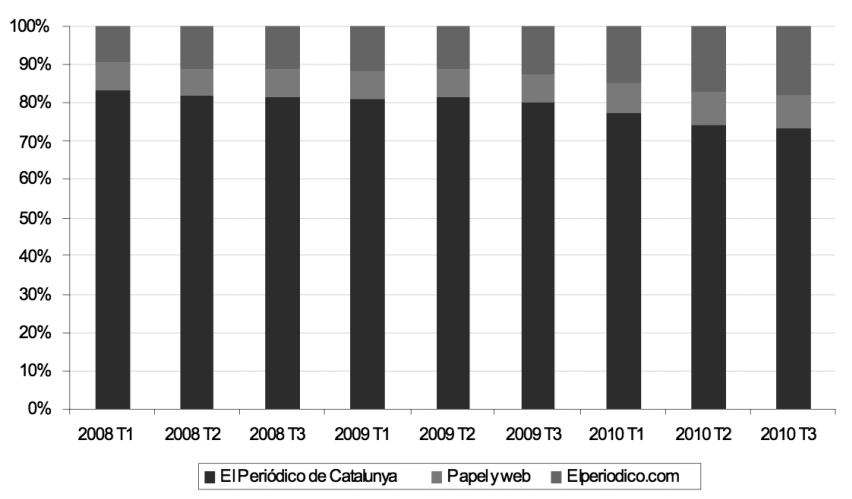

\begin{tabular}{|l|l|}
\hline & Variación 2008 \\
\hline El Periódico & $-4,10 \%$ \\
\hline ElPeriodico.com & $72,31 \%$ \\
\hline Leen ambos & $37,20 \%$ \\
\hline
\end{tabular}


Tabla I. Muestras escogidas para el análisis de los datos

\begin{tabular}{|l|l|l|}
\hline $\begin{array}{l}\text { Muestra: lectores habituales de la } \\
\text { edición on-line }\end{array}$ & Vínculo habitual & Vínculo mínimo \\
\hline $\begin{array}{l}\text { Frecuencia de compra de la edición } \\
\text { impresa }\end{array}$ & A diario o 2-5 veces a la semana & Una vez a la semana \\
\hline
\end{tabular}

Tabla II. Lugares de lectura para la edición impresa y la edición on-line

\begin{tabular}{|l|l|l|l|l|}
\hline \multirow{2}{*}{} & \multicolumn{2}{|c|}{ Lectura edición impresa } & \multicolumn{2}{c|}{ Lectura edición on-line } \\
\cline { 2 - 5 } & vínculo habitual & vínculo mínimo & vínculo habitual & vínculo mínimo \\
\hline En casa & $87,51 \%$ & $86,83 \%$ & $68,69 \%$ & $73,39 \%$ \\
\hline En el trabajo & $21,40 \%$ & $19,81 \%$ & $56,60 \%$ & $46,44 \%$ \\
\hline En el transporte & $12,88 \%$ & $11,85 \%$ & & \\
\hline En bares & $30,17 \%$ & $27,48 \%$ & & \\
\hline
\end{tabular}

Tabla III. Momentos de lectura para la edición impresa y para la edición digital

\begin{tabular}{|l|l|l|l|l|}
\hline \multirow{2}{*}{} & \multicolumn{2}{|c|}{ Momento lectura edición impresa } & \multicolumn{2}{c|}{ Momento lectura edición on-line } \\
\cline { 2 - 5 } & vínculo habitual & vínculo mínimo & vínculo habitual & vínculo mínimo \\
\hline Mañana & $76,14 \%$ & $72,42 \%$ & $74,66 \%$ & $51,97 \%$ \\
\hline Tarde & $20,60 \%$ & $21,29 \%$ & $27,21 \%$ & $24,47 \%$ \\
\hline Noche & $30,35 \%$ & $29,66 \%$ & $25,31 \%$ & $39,40 \%$ \\
\hline
\end{tabular}


Tabla IV. Tiempo de dedicación a la lectura de la edición impresa y de la edición on-line

\begin{tabular}{|l|l|l|l|l|}
\hline \multirow{2}{*}{} & \multicolumn{2}{|c|}{ Tiempo de lectura de la edición impresa } & \multicolumn{2}{c|}{ Tiempo de lectura de la edición on-line } \\
\cline { 2 - 5 } & vínculo habitual & vínculo mínimo & vínculo habitual & vínculo mínimo \\
\cline { 1 - 4 } Menos de 15 minutos & $19,41 \%$ & $30,67 \%$ & $38,59 \%$ & $51,67 \%$ \\
\cline { 1 - 4 } Entre 15 y 30 minutos & & & $47,99 \%$ & $40,20 \%$ \\
\hline $\begin{array}{l}\text { Entre 30 minutos y una } \\
\text { hora }\end{array}$ & $64,44 \%$ & $56,00 \%$ & $11,82 \%$ & $6,86 \%$ \\
\hline Entre una y dos horas & $14,47 \%$ & $12,00 \%$ & $1,39 \%$ & $0 \%$ \\
\hline Más de 2 horas & $1,69 \%$ & $1,33 \%$ & $0,22 \%$ & $1,26 \%$ \\
\hline
\end{tabular}

Tabla V. Variación del tiempo dedicado a cada una de las ediciones durante el fin de semana

\begin{tabular}{|l|l|l|l|l|}
\hline \multirow{2}{*}{} & \multicolumn{2}{|c|}{$\begin{array}{c}\text { Lectura edición impresa durante el fin } \\
\text { de semana }\end{array}$} & \multicolumn{2}{c|}{$\begin{array}{c}\text { Lectura edición on-line durante el fin } \\
\text { de semana }\end{array}$} \\
\cline { 2 - 5 } & vínculo habitual & vínculo mínimo & vínculo habitual & vínculo mínimo \\
\hline Más tiempo & $78,07 \%$ & $75,21 \%$ & $14,47 \%$ & $26,67 \%$ \\
\hline El mismo tiempo & $14,76 \%$ & $22,21 \%$ & $35,26 \%$ & $20,00 \%$ \\
\hline Menos tiempo & $6,17 \%$ & $2,58 \%$ & $28,33 \%$ & $30,67 \%$ \\
\hline Ninguno & $1,00 \%$ & $0 \%$ & $21,94 \%$ & $22,67 \%$ \\
\hline
\end{tabular}


Tabla VI. Valoraciones de los lectores sobre la edición impresa

\begin{tabular}{|c|c|c|}
\hline & vínculo habitual & vínculo mínimo \\
\hline Me gustan los reportajes a fondo que analizan un tema en concreto & 4,05 & 4,04 \\
\hline Lo más importante en el periódico es que sea actual & 4 & 4,31 \\
\hline Compro La Vanguardia porque es más objetiva que otras cabeceras & 3,56 & 3,82 \\
\hline Me identifico con la línea editorial de La Vanguardia & 3,51 & 3,76 \\
\hline El periódico es más objetivo y veraz que otros medios de información & 3,36 & 3,78 \\
\hline Un periódico debe ser entretenido y divertido & 3,25 & 3,49 \\
\hline Normalmente me intereso solo por algunas secciones en concreto & 3,29 & 3,39 \\
\hline Prefiero las noticias informativas antes que los artículos de opinión & 3,12 & 3,13 \\
\hline Lo que más me interesa son las columnas de opinión & 2,93 & 3,15 \\
\hline Las noticias locales son más importantes que las noticias internacionales & 2,78 & 2,89 \\
\hline Normalmente ojeo los titulares pero no me paro a leer a fondo las noticias & 2,63 & 2,84 \\
\hline Compro el periódico cuando me interesa un tema en concreto & 2,05 & 1,68 \\
\hline $\begin{array}{l}\text { Solo compro el periódico cuando viene acompañado de un suplemento que me } \\
\text { gusta }\end{array}$ & 1,58 & 1,53 \\
\hline Participo frecuentemente enviando cartas al director & 1,39 & 1,43 \\
\hline
\end{tabular}


Tabla VII. Valoraciones de los lectores sobre la edición on-line

\begin{tabular}{|l|l|l|}
\hline & vínculo habitual & vínculo mínimo \\
\hline Aunque leo la versión digital me gusta más el periódico de papel & 3,8 & 4,24 \\
\hline La versión digital es de más fácil consulta & 3,43 & 3,01 \\
\hline $\begin{array}{l}\text { Prefiero LaVanguardia.com porque aporta información más actualizada que la } \\
\text { versión en papel }\end{array}$ & 3,23 & 3,06 \\
\hline Leo la versión digital porque es gratis & 3,19 & 3,01 \\
\hline $\begin{array}{l}\text { Leo la versión digital porque me aporta la misma información que el papel de manera } \\
\text { más fácil }\end{array}$ & 2,95 & 2,73 \\
\hline Leo la versión digital porque puedo hacerlo mientras trabajo & 3,17 & 2,71 \\
\hline Leo tanto el papel como el digital porque son cosas distintas & 3,13 & 3,19 \\
\hline La versión digital es más entretenida que la impresa & 2,52 & 2,57 \\
\hline Lo que más me gusta de la versión digital es poder opinar & 2,35 & 2,36 \\
\hline $\begin{array}{l}\text { La información del periódico de papel me da más confianza que la de la versión } \\
\text { digital }\end{array}$ & 2,34 & 2,78 \\
\hline La información de la versión digital es más objetiva & 2,07 & 2,11 \\
\hline
\end{tabular}


Tabla VIII. Actividades realizadas en el site

\begin{tabular}{|l|l|l|l|}
\hline Categoría & Actividades & Vínculo habitual & Vínculo mínimo \\
\hline \multirow{2}{*}{\begin{tabular}{l} 
Búsqueda de $\begin{array}{l}\text { información } \\
\text { actualizada }\end{array}$ \\
\cline { 2 - 4 }
\end{tabular}} & Leer los titulares de la portada & $74.5 \%$ & $45.0 \%$ \\
\cline { 2 - 4 } $\begin{array}{l}\text { Búsqueda o } \\
\text { recuperación de } \\
\text { información } \\
\text { específica }\end{array}$ & Consultar información de última hora & $72.7 \%$ & $66.9 \%$ \\
\cline { 2 - 4 } & Buscar información específica & $57.7 \%$ & $47.8 \%$ \\
\cline { 2 - 4 } & Buscar información en la Hemeroteca & $32.5 \%$ & $48.2 \%$ \\
\cline { 2 - 4 } & Documentarme & $32,60 \%$ & $47.1 \%$ \\
\cline { 2 - 4 } & Enviar noticias a amigos & $31.7 \%$ & $36.3 \%$ \\
\cline { 2 - 4 } Participación & Comentar noticias & $32.9 \%$ & $35.2 \%$ \\
\cline { 2 - 4 } & Participar en foros & $8.9 \%$ & $17.6 \% \%$ \\
\cline { 2 - 4 } & Otros & $8.8 \%$ & $12.0 \%$ \\
\hline
\end{tabular}

\section{Referencias bibliográficas}

Armentia, J. I., Caminos, J. M., Elexgaray, J., Martin, F., \& Merchán, I. (2000): El diario digital: Análisis de los contenidos textuales, aspectos formales y publicitarios. Barcelona: Bosch.

Beaudoin, E.; Thorston, E. (2002): “A marketplace theory of media use”, Mass Communication \& Society, vol. 5, pp. 241262.

Bucy, E. (2003): “Media credibility reconsidered: Synergy effects between on-air and online news”, Journalism \& Mass Communication Quarterly, vol. 80, pp. 247-265.

Cabrera, M. A. (coord.) (2010): Evolución tecnológica y cibermedios. Zamora: Comunicación social.

Callejo, J. (2001): Investigar las audiencias. Un análisis cualitativo. Barcelona: Paidós.

Canga, J., Coca, C., Martínez, E., Cantalapiedra, M. J., y Martínez, L. (2000): Diarios digitales. Apuntes sobre un nuevo medio. Bilbao: Universidad del País Vasco.

$36\left|n^{\circ} 14\right|$ doxa.comunicación 
Chung, D. S. (2008): “Interactive features of online newspapers: Identifying and predicting use of engaged readers”, Journal of Computer Mediated Communication, vol. 13 (3), pp. 658-679.

Chung, D. S.; \& Yoo, Y. (2006): “Online user motivations and use of interactive features on an online news site: A uses and gratifications approach”, Conference Papers International Communication Association, pp. 1-36.

De Waal et al. (2005): "Online Newspapers: A Substitute or Complement for Print Newspapers and Other Information Channels?”, Communications, vol. 30, pp. 55-72.

Dimmick, J.W. (2003): Media competition and coexistence: The theory of the niche. Wahwam: Lawrence Erlbaulm.

Dutta-Bergman, M. J. (2004): "Complementarity in consumption of news types across traditional and new media”, Journal of Broadcasting \& Electronic Media, vol. 48 (1), pp. 41-60.

Fernández, A. (2011). “Diarios para el ipad, nuevas oportunidades para el periodismo digital”, en J. J.Verón, y F. Sabés (eds.): La investigación para el periodismo digital. algunos trabajos desde el ámbito universitario. Congreso de Periodismo Digital (Huesca).

Fidler, R. F. (1997): Mediamorfosis: Comprender los nuevos medios. Granica: Buenos Aires.

Flavián, C., y Gurrea, R. (2006): “The choice of digital newspapers: Influence of reader goals and user experience”, Internet Resarch, 16 (3), pp. 231-247.

Gabardo, J. A. (2010): “Internet en medio de los medios”, Seminario de medios en Madrid, AIMC.

Kiousis, S. (2001): "Public trust or mistrust? Perceptions of media credibility in the information age", Mass Communication \& Society, vol. 4, pp. 381-404.

Lee, P.; Leung, L. (2006): "Assessing the displacement effects of the Internet”, Telematics and Informatics, vol. 25, pp. 145155.

Livingstone, S. (1999): “New media, new audiences?”, New media and society, New media and society, vol. 1 (1). pp. 59-66.

Meyen, M. (2004): Mediennutzung. Konstanz: UVK.

Mitchelstein, E.; Boczkowski, P. (2010): “Online news consumption research: An assessment of past work and an agenda for the future”, New Media \& Society, vol. 12 (7), pp. 1085-1102.

McQuail, D. (1997): Audience analysis. Londres: Sage Publications.

- (2000): Introducción a la Teoría de la Comunicación de Masas. Paidós: México.

Nguyen, A., y Western, M. (2006): “The complementary relationship between the internet and traditional mass media: The case of online news and information", Information Research, vol. 11 (3), pp. 11-13.

Oyedeji, T. (2007): “The Relation Between the Customer-Based Brand Equity of Media Outlets and Their Media Channel Credibility", The International Journal on Media Management, vol. 9 (3), pp. 116-125. 
Palmgreen, P.; Rayburn, J.D. (1985): “An expectancy-value approach to media gratifications”, en Rosengren, K. E.; Wenner, L. A. (1985): Media gratifications research: Current perspectives. USA: Sage Publications.

Robinson, J. et al. (2000): “Mass Media Use and Social Life Among Internet Users”, Social Science Computer Review, vol. 18 (4), pp. 490-501.

Ruggiero, T. E. (2000): “Uses and gratifications theory in the 21st century", Mass Comunication \& Society, vol. 3 (1), pp. 3 37.

Stempel et al. (2000): "Relation of Growth of Use of the Internet to Changes in Media Use from 1995 to 1999", Journalism and Mass Communication Quarterly, vol. 77, pp. 71-79. 